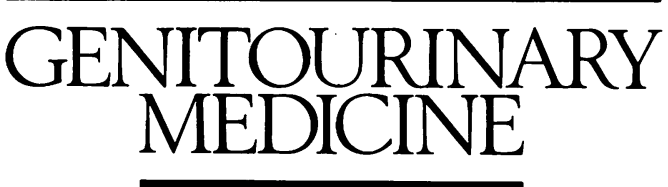

Editorial

\title{
How can industry, academia, public health authorities and the Sexually Transmitted Diseases Diagnostics Initiative (SDI) work together to help control sexually transmitted diseases in developing countries?
}

It is estimated that during 1997 there will be over 300 million new cases of sexually transmitted diseases due to one or more of the following: gonorrhoea, chlamydia, syphilis, chancroid. About $85 \%$ of these will occur in developing countries. All of these diseases are diagnosable and treatable. Because of ulcerations caused by Treponema pallidum (syphilis) and Haemophilus ducreyi (chancroid) and inflammation due to Neisseria gonorrhoeae or Chlamydia trachomatis, these infections facilitate and enhance transmission of HIV in both men and women. ${ }^{1-3}$ Rapid diagnosis and treatment of STDs has become one of the primary strategies for prevention of sexual transmission of HIV.

Existing diagnostic tests for STDs are sensitive and specific, but because of costs, complexity, time to get a result and a need for local laboratory infrastructure, their use is not practical at peripheral health facilities in many developing countries. Because effective control of STDs requires diagnosis and treatment at the point of first encounter, with minimal delay between diagnosis and treatment, UNAIDS and WHO recommend syndromic management of STDs. This requires the identification of persons with collections of signs and symptoms (STD syndromes) and the provision of treatment for all the sexually transmitted infections commonly associated with that syndrome in that particular geographic setting. Because the syndromic approach cannot differentiate specific infections within syndromes, over-treatment occurs with relatively expensive drugs. In addition to adding to the financial burden of health care, such over-treatment may contribute to the development of antibiotic-resistant strains. Since the majority of gonorrhoeal and chlamydial infections in women are asymptomatic they cannot be diagnosed by a syndromic approach. The majority of these infections will therefore not be treated, resulting in sequelae and continued STD and HIV transmission.

The Sexually Transmitted Disease Diagnostics Initiative (SDI) was formed in November of 1990 by an international group of physicians, scientists and other health care workers interested in diagnosing and controlling STDs. The original mission of the SDI was to identify or develop affordable diagnostic tools appropriate for use in resource-limited settings and to introduce these tools into STD prevention and control programs. This means tests that are sensitive, specific, simple, stable and inexpensive. From 1990-93 the SDI Secretariat was in Seattle as part of the
Program for Appropriate Technology in Health (PATH) and the SDI steering committee was chaired by Seth Berkley (Rockefeller Foundation). There were three original working groups chaired by King Holmes (Syndromic Diagnosis), Steve Morse (Syphilis and Gonorrhoea) and Julius Schachter (Chlamydia). Because more international representation and exposure was needed the WHO offered to serve as the secretariat, thus the SDI became an Initiative under the Global Programme for AIDS (GPA) in 1994 and PATH became a Participating Centre (PC). On January 1, 1996 UNAIDS began serving as the SDI secretariat. Under the existing structure the SDI receives donor support as well as guidance on finance and management from a Committee of Interested Parties (CIP). A Steering Committee with international representation, makes technical and scientific recommendations to the SDI, mainly through the administration of a grants program. Since 199473 letters of intent from 23 countries were received by the SDI. Twenty-three full proposals were invited and eight were approved for funding. Four of the projects involve the development of new tests for chlamydia, three for gonorrhoeae, two for chancroid, and there is one joint project on syphilis. The total amount of money allocated for these projects is $\$ 850000$ US over a three year period. As each project becomes funded, the investigator and institution form a PC. The SDI accepts letters of intent at any time and schedules two full proposal deadlines 1 January and 1 July.

In 1990 , the global budget for health care exceeded 1.7 trillion US dollars or $8 \%$ of total world product. Despite representing less than one quarter of the world population, industrialised countries accounted for approximately $90 \%$ of this total health care expenditure. Even so, developing countries spent a substantial total of 170 billion dollars on health care in 1990. Current STD diagnostic needs for 16 selected countries with a total of the world population of 5.2 billion inhabitants, are estimated to be at least 326 million STD tests. This would be only for diagnosing and differentiating symptomatic gonorrhoea and chlamydial infections, syphilis and chancroid; and for screening women attending antenatal clinics for gonorrhoea, chlamydial infection and syphilis. Of the $326 \mathrm{M}$ tests, $5 \%$ or 16 million would be required by industrialised countries and the remaining $95 \%$ or 310 million, representing significant market potential, and the majority of need, 
would be required by developing countries. The absolute expenditure on health care in both industrialised and developing country markets is set by economic conditions and social and political policy. Nonetheless, the end result of use of STD diagnostic tests meeting the SDI objectives would be an increase in comprehensive disease management efficiencies in all countries where the test was made available. This increase in efficiency could be expected to encourage greater expenditures on STDs as rapid diagnosis and treatment make their management and case finding become more effective and affordable. The increase in sales of test and drugs for STD management and case finding would thus provide positive economic and social returns in developing and industrialised countries alike. Donor governments and the World Bank have identified early diagnosis and treatment of STD as a major priority in order to prevent STD sequelae and decrease the rate of HIV transmission. As a result they are currently financing the purchase of antibiotics to treat STDs in over 25 developing countries. Were an easy-to-perform, cost effective STD diagnostic test available it too could be financed as a means of increasing "value for money".

Although there are many constraints to product development and market penetration for developing countries, collaboration between industry and the SDI should be able to reduce or eliminate most of them. The SDI has established a library of fully characterised clinical specimens to be used by donors and participating centres in industry or academia. Through its newsletter Global Access to STD Diagnostics instructions for collection and handling of specimens and proper evaluation of chlamydia diagnostics have been published. The SDI can facilitate validation studies and field trials in developing or developed countries. The SDI has the capacity to establish ad hoc working groups or public forums on technology, regulation, liability, business or marketing issues in any part of the world. Through UNAIDS and WHO the SDI can provide comprehensive country or regional needs and market information. This relationship can facilitate easier country advocacy, reduced import barriers and bulk procurement and distribution. The UNAIDS/SDI relationship also provides easier access to World Bank and non-governmental services goals.

Industry has an outstanding record regarding public sector collaboration to achieve common goals. Many of these public-private sector partnerships have resulted in the discovery of diagnostic tests and/or pharmaceuticals which have eradicated disease and increased quality of life. Examples are the Onchocerciasis Control Programme in Saharan Africa which has made Ivermectin available since 1974, and the polio and measles programmes fostered by Rotary International. Industry can share or exchange technical and scientific information confidentially with the SDI, or participate through cross-licensing or other mechanisms with other companies collaborating with SDI. Industry can contribute financially or otherwise to the activities of the SDI. By doing this a company may be capable of securing a place in the CIP beside non-industry donors, becoming a full participant in the process. This could lead to SDI funded joint research projects between industry and the other SDI components at stages of product development, evaluation, production or distribution.

There are certainly many incentives to achieve the goals is of the SDI. For researchers the Rockefeller Foundation $\vec{\circ}$ has established a 1 million dollar prize to be awarded to the scientist or team, capable of constructing a rapid sensi- $\vec{\sigma}$ tive, specific, simple, stable and inexpensive assay. The SDI provides research funding and an easy access to pedigreed clinical specimens as well as guidelines and developing country sites for effective evaluations. For industry, the markets are there and the challenge is out. Combined $\frac{9}{\partial}$ with an increased level of social responsibility, and enhanced by the importance of STD diagnosis and treat- $\mathbb{D}$ ment in AIDS prevention, there is a synergistic environment for the SDI, governments, traditional donors and the diagnostics and pharmaceutical industries to join forces, maximise resources and significantly contribute to the control of STD.

Chairman, SDI Steering Committee,

MAX A CHERNESKY, PHD

St foseph's Hospital-McMaster University,

50 Charlton Avenue East,

Hamilton, Ontario, Canada L8N $4 A 6$

1 Laga M, Nzila N, Goeman J. The interrelationship of sexually transmitted diseases and HIV infection: implications for the control of both epidemics in Africa. AIDS 1991;5(suppl 1):S55-63.

2 Wasserheit JN. Epidemiological synergy: interrelationships between human immunodeficiency virus infection and other sexually transmitted diseases. Sex Transm Dis 1992;19:61-77.

3 Laga M, Manoka A, Kivuvu M, Malele B, Tuliza M, Nzila N, et al. Nonulcerative sexually transmitted diseases as risk factors for HIV-1 transmission in women: results from a cohort study. AIDS 1993;7:95-102. 